\title{
A study of paediatric dermatoses in tertiary care center, Gandhinagar, Gujarat
}

\author{
Kalpesh M Prajapati ${ }^{1}$, Bela Padhiar,"* \\ ${ }^{\mathbf{1}}$ Resident, ${ }^{\mathbf{2}}$ Associate Professor and HOD, Dept. of Dermatology, GMERS Medical College, Gandhinagar, Gujarat, India
}

*Corresponding Author:

Email: drbelapadhiar@gmail.com

\begin{abstract}
Introduction: Pediatric dermatology is an important branch of dermatology that deals with the diagnosis, treatment, and prevention of skin diseases occurring in infancy, childhood, and adolescence.

Aims: The present study was conducted to find out the pattern and prevalence of various dermatosis among the paediatric age group.

Materials and Methods: This is prospective, cross sectional study. A total 2456 patients were recruited in 1-14 year of age, who presented with dermatosis in dermatology clinic of GMERS medical college, Gandhinagar. The study was conducted from March 2016 to February 2017(12 Months), and diagnosis was made based on clinical examination and necessary investigation if required.

Results: Males were outnumbered female in present study with ratio of 1.45:1. The majority of patients were in the age group of $>6-14$ year (44.09\%). Infections and infestations were most common constituting $65.19 \%$ followed by eczema and dermatitis group of disorder constituting $12.02 \%(n=324)$. Appendageal disorder constitutes $6.42 \%(n=173)$ followed by cutaneous vascular response $(4.27 \%)$, nutritional dermatosis $(2.67 \%)$, pigmentary disorder $(2.15 \%)$, keratinization disorder $(2.0 \%)$ and papulosquamous disorder $(1.89 \%)$. Least common disorder include nevi, genodermatoses, collagen vascular disorder and vesiculobullous diorde.

Conclusion: This study helps us to know the prevalence of skin diseases in pediatric age group and thus we can take adequate measures for their prevention and treatment in this region.
\end{abstract}

Keywords: Paediatric, Dermtoses, Infections and Infestations.

\section{Introduction}

Paediatric dermatology is an important branch of dermatology that deals with the diagnosis, treatment, and prevention of skin diseases occurring in infancy, childhood, and adolescence. ${ }^{1}$ Skin is the largest organ of human body. There are various differences in the child and adult skin. Anatomically, it is thinner less cornified and less hairy while physiologically, sweat and sebaceous secretion are scantier.

Dermatological problems account for about $30 \%$ of primary and secondary reasons for pediatric clinic visits and $30 \%$ of all visits to dermatologists involve patients of pediatric age group. ${ }^{2}$

The incidence of pediatric dermatoses is showing upward trend gradually and need exists to pay more attention to this problem. ${ }^{3}$ The incidence of skin diseases among children in various parts of India has ranged from $8.7 \%$ to $38.8 \%$ in different studies usually school based surveys. ${ }^{4}$

There are many risk factor involve in prevelance and pattern of various dermatoses in paediatric population such as ecological, geographical, climatic exposure, season, personal hygiene, large family size or overcrowding, poverty, malnutrition, illiteracy, Socioeconomic status. External environment, excess population and over-crowding are important risk factor for development of disease in urban area. They can be transitory or chronic, recurrent and although they are rarely lethal, they may be associated with significant morbidity and psychological impact as well as an implication in their development. Traditional tattoos, customs, habits, lack of health care facilities, lack of sanitation and constant exposure to trauma are responsible for recurrent and chronicity of various dermatoses. Most of cutaneous disease that results from genetic abnormalities have onset in the paediatric age group.

Diagnosis of pediatrics dermatoses require separate view from adult dermatoses because it require special skills, important difference in anatomical structure, physiological difference and difference in clinical presentation, management and prognosis.

Skin diseases in children are encountered frequently and their characterization is essential for the preparation of academic, research and health plans. ${ }^{5}$ Studies of pediatric population which constitutes the cornerstone of the community can play an important role in determining the policies of protective medicine and public health. ${ }^{6}$ This study also serves as a contribution for future survey.

\section{Materials and Methods}

This was a "Prospective, cross-sectional observation" single centre study carried out in dermatology outpatient department of GMERS Medical College, Gandhinagar, Gujarat, a tertiary care teaching hospital in western India. The study protocol was presented and approved by an IEC (22/2015). Patients were explained clearly about the nature and purpose of the study in the language they understood. Written informed consent and photographs were obtained for medical records before enrolling the patients for the 
study. Total 2456 cases were enrolled in our study. A detailed general, systemic and cutaneous examination followed by relevant investigations was carried out. The findings were recorded in a proforma for analysis and interpretation of data.

Inclusion Criteria: Age group below 14 (neonate, infant, child), both male and female, Include both indoor and outdoor patients, those who willing for examination of their child was included and also included skin ADR patients.

Exclusion Criteria: Guardian not willing to participate in study group all the eligible cases attending skin \& VD OPD, civil Hospital, GMERS Medical College Gandhinagar during the academic time period (2 years) were included for the study with written consent. Detailed history including age at the time of attending clinic, sex, History regarding onset, duration, symptoms, severity and progression of lesions history of atopy was taken. Family history of similar complaints was also taken. Each patient was subjected to detailed history taking and clinical examination (both general and dermatological examination) done. Relevant investigations pertaining to the clinical presentation such as complete blood picture, erythrocyte sedimentation rate, renal function test and liver function test, specific investigation such as smears (microscopic examination of scraping for superficial fungal, wood's light examination and infection tissue smear stained with tzanck for viral infection and immune bullous disorder) and biopsy were carried out as and when required. A final diagnosis was then made based on the clinical features and investigations. All variable (age, gender, skin disease) were expressed in frequency and percentage. $\mathrm{P}<0.05$ was considered as statistical significance.

\section{Results}

Total 2456 number of patients was enrolled in present study form March 2016 to February 2017 (12 Months). Highest number of patients ( $\mathrm{n}=1083,44.09 \%)$ were in >6-14 years of age group followed by $>1-6$ years of age group $(\mathrm{n}=1021,41.57 \%)$. Male preponderance was seen with Male: Female ratio of 1.43:1. Among neonate, infant, preschool child and school child, it was $2: 1,1.3: 1,1.3: 1$ and 1.6:1 respectively. (Table 1)

Among neonate, the physiological skin disease erythema toxicum neonatorum $(\mathrm{n}=11)$ was most common whereas among the pathological skin disease miliria $(n=20)$ was most common followed by candidiasis $(\mathrm{n}=10)$. (Table 2$)$

Among children of $>1$ month of age group, infectious group of disorder were most prevalent amongst all disorder with total number 1757 (65.19\%) followed by eczema and dermatitis group of disorder with total number of $324(12.02 \%)$. Male predominance seen in all group of disorder except for pigmentary disease where female predominance seen where-as nevi and other developmental disorder and keratinizing disorder had equal prevalence. (Table 3)

Bacterial infection were most common $(n=791$, $29.33 \%$ ) followed by disease caused by arthropod with total number of $492(18.25 \%)$. There was marginal difference of prevalence between fungal and viral infection. There was male preponderance seen in all age groups and among all infectious diseases. (Table 4) As shown in (Table 4), most common disease was impetigo $(\mathrm{n}=295,10.94 \%)$ followed by decreasing order were boil/carbuncle/ pyoderma> periporitis> scalp pyoderma> furuncle> cellulitis with all disease had male preponderance. Amongst infant, impetigo was more common in female. Boil/ carbuncle were more common among school child with total number of 112 as compared to impetigo $(\mathrm{n}=78)$.

As depicted in (Table 4), most common viral disease was Chicken pox $(02.41 \%)$ followed by Warts $(01.78 \%)$, Pityriasis Rosea (01.37\%), molluscum contagiosum $(01.22 \%)$, hand foot mouth disease $(01.07 \%)$. Herpes simplex and herpes zoster were least common viral disorder. Amongst infant, hand foot mouth disease was more common $(n=08)$. Preschool child were more affected by molluscum contagiosum $(n=26)$ followed by hand foot mouth disease $(n=19)$. Chicken pox $(n=41)$ was more common among the school going child followed by warts $(n=30)$ and pityriasis rosea $(n=27)$. Female predominance was seen in chicken pox amongst school going child and in warts among preschool child.

Most common disease among eczematous group of disorder was atopic disease $(n=81,03.00 \%)$ followed by eczema (discoid, lichenoid, nummular, dishydrotic etc.) $(02.82 \%)$, pityriasis alba $(02.55 \%)$, seborrheic dermatitis $(02.48 \%)$. (Table 5)

Amongst the nevus and other developmental disorder, haemangioma was most common with total number of 13 followed by nevus depigmentosus $(n=06)$, congenital melanocytic nevi $(n=05)$, verrucous epidermal nevus $(\mathrm{n}=04)$, nevus of ito/ota $(\mathrm{n}=3)$. Vitiligo $(n=42)$ was most common disease amongst pigmentary disorder and among all age group female were more common than male.

Amongst the papulosquamous disorder, lichen planus $(n=21)$ followed by psoriasis $(n=12)$, lichen striatus $(n=10)$ and lichen nitidus $(n=08)$. Lichen planus and psoriasis more common among school child with total number of 14 and 09 respectively. Psoriasis was more common in female $(n=7)$ then male $(n=5)$. Lichen striatus was more common among preschool child $(\mathrm{n}=08)$.

Most common disease among the keratinizing disorder was keratoderma $(n=37)$ followed by icthyosis $(\mathrm{n}=08)$, keratosis pillaris $(\mathrm{n}=07)$ and lichen spinulosa $(n=02)$. Keratosis pilaris was more common amongst school child. Female were more common amongst keratoderma. 
Hundred and twenty one $(n=121)$ were of Miliaria, followed by alopecia aerata $(n=20)$, acne $(n=18)$, milia $(n=10)$ and least common was hyperhidrosis $(n=04)$. Milia were more common in preschool child whereas acne was more common among school child. Alopecia aerata was more common among female $(n=11)$ then male $(n=9)$.
Neurofibromatosis was most common amongst all genodermatoses followed by equal prevalence of wardernburg syndrome, xeroderma pigmentosus, anhdrotic ectodermal dysplasia, tuberous sclerosis complex.

Table 1: Gender distribution according to age group

\begin{tabular}{|l|c|c|c|c|}
\hline \multicolumn{1}{|c|}{ Age } & Male & Female & Total & Percentage \\
\hline$<1$ month (neonate) & 37 & 18 & 55 & $02.23 \%$ \\
\hline$>$ 1month-1 year (infant) & 168 & 129 & 297 & $12.09 \%$ \\
\hline$>$ 1year-6year(preschool child) & 578 & 443 & 1021 & $41.57 \%$ \\
\hline$>$ 6year-14year (school child) & 671 & 412 & 1083 & $44.09 \%$ \\
\hline Total & 1454 & 1002 & 2456 & $100 \%$ \\
\hline Percentage & $59.20 \%$ & $40.80 \%$ & $100 \%$ & \\
\hline
\end{tabular}

Table 2: Pattern O various disorders in neonate ( $<1$ month)

\begin{tabular}{|c|c|c|c|}
\hline Category & Disease & No of Neonate & Percentage \\
\hline Physiological & Vernix caseosa & 2 & $03.33 \%$ \\
\hline & Sebaceous hyperplasia & 2 & $03.33 \%$ \\
\hline & Milia & 3 & $05.00 \%$ \\
\hline & Cutis marmorta & 1 & $01.67 \%$ \\
\hline & Mongolian spot & 3 & $05.00 \%$ \\
\hline & Erythematoxicum neonatorum & 11 & $18.33 \%$ \\
\hline Pathological & Cradle cap & 2 & $03.33 \%$ \\
\hline & Miliria & 20 & $33.33 \%$ \\
\hline & Periporitis & 3 & $05.00 \%$ \\
\hline & Impetigo & 3 & $05.00 \%$ \\
\hline & Candidiasis & 10 & $16.67 \%$ \\
\hline & & 60 & $100 \%$ \\
\hline
\end{tabular}

Table 3: Pattern of various disorders in $>1$ month of age group

\begin{tabular}{|c|c|c|c|c|c|c|c|c|c|c|}
\hline \multirow[t]{3}{*}{ Dermatosis } & \multicolumn{8}{|c|}{ Age distribution } & \multirow{3}{*}{$\begin{array}{c}\text { Grand } \\
\text { total }\end{array}$} & \multirow{3}{*}{ Percentage } \\
\hline & \multicolumn{2}{|c|}{$\begin{array}{l}1 \text { month-1 } \\
\text { year }\end{array}$} & \multicolumn{2}{|c|}{$>1-6$ year } & \multicolumn{2}{|c|}{$>6-14$ year } & \multicolumn{2}{|c|}{ Total } & & \\
\hline & $\mathrm{M}$ & $\mathrm{F}$ & $\mathrm{M}$ & $\mathrm{F}$ & $\mathrm{M}$ & $\mathrm{F}$ & $\mathrm{M}$ & $\mathrm{F}$ & & \\
\hline Infectious disease & 115 & 91 & 460 & 321 & 498 & 272 & 1073 & 684 & 1757 & $65.19 \%$ \\
\hline Eczema and dermatitis & 30 & 20 & 62 & 64 & 85 & 63 & 177 & 147 & 324 & $12.02 \%$ \\
\hline $\begin{array}{l}\text { Nevi and other } \\
\text { developmental disoder }\end{array}$ & 06 & 07 & 08 & 06 & 05 & 07 & 19 & 19 & 38 & $01.41 \%$ \\
\hline Pigmentary disoder & 03 & 03 & 05 & 08 & 20 & 19 & 28 & 30 & 58 & $02.15 \%$ \\
\hline Nutritional disorder & 02 & 06 & 19 & 13 & 19 & 13 & 40 & 32 & 72 & $02.67 \%$ \\
\hline $\begin{array}{l}\text { Papulosquamous } \\
\text { disoder }\end{array}$ & 00 & 00 & 11 & 12 & 17 & 11 & 28 & 23 & 51 & $01.89 \%$ \\
\hline Keratinization disorder & 02 & 00 & 09 & 13 & 16 & 14 & 27 & 27 & 54 & $02.00 \%$ \\
\hline $\begin{array}{l}\text { Vesiculo bullous } \\
\text { disorder }\end{array}$ & 00 & 00 & 00 & 00 & 00 & 01 & 00 & 01 & 01 & $00.04 \%$ \\
\hline Appendageal disorder & 14 & 07 & 48 & 38 & 31 & 35 & 93 & 80 & 173 & $06.42 \%$ \\
\hline $\begin{array}{l}\text { Collagen vascular } \\
\text { disorder }\end{array}$ & 00 & 00 & 00 & 00 & 02 & 03 & 02 & 03 & 05 & $00.20 \%$ \\
\hline Genodermatosis & 00 & 00 & 01 & 03 & 07 & 05 & 08 & 08 & 16 & $00.59 \%$ \\
\hline $\begin{array}{l}\text { Cutaneous vascular } \\
\text { response }\end{array}$ & 07 & 04 & 19 & 21 & 40 & 24 & 66 & 49 & 115 & $04.27 \%$ \\
\hline \multirow[t]{2}{*}{$\begin{array}{l}\text { Miscellaneous } \\
\text { disorder }\end{array}$} & 01 & 01 & 04 & 02 & 17 & 06 & 22 & 09 & 31 & $01.15 \%$ \\
\hline & & & & & & & & & 2695 & $100 \%$ \\
\hline
\end{tabular}


Table 4: Pattern of infectious disorders in $>1$ month of age group

\begin{tabular}{|c|c|c|c|c|c|c|c|c|c|c|c|}
\hline \multirow[t]{3}{*}{ Category } & \multirow[t]{3}{*}{ Disease } & \multicolumn{6}{|c|}{ Age group distribution } & \multicolumn{2}{|c|}{ Total } & \multirow{3}{*}{$\begin{array}{c}\text { Grand } \\
\text { Total }\end{array}$} & \multirow[t]{2}{*}{ Percentage } \\
\hline & & \multicolumn{2}{|c|}{$\begin{array}{l}1 \text { month } \\
\text { to } 1 \text { year }\end{array}$} & \multicolumn{2}{|c|}{$\begin{array}{c}>1 \text { year to } \\
6 \text { year }\end{array}$} & \multicolumn{2}{|c|}{$\begin{array}{c}>6 \text { year to } \\
14 \text { year }\end{array}$} & & & & \\
\hline & & $\mathrm{M}$ & $\mathrm{F}$ & $\mathrm{M}$ & $\mathrm{F}$ & $\mathrm{M}$ & $\mathrm{F}$ & $\mathrm{M}$ & $\mathrm{F}$ & & \\
\hline \multirow[t]{7}{*}{ Bacterial } & Impetigo & 18 & 22 & 106 & 73 & 45 & 31 & 169 & 126 & 295 & $10.94 \%$ \\
\hline & Periporitis & 05 & 07 & 59 & 37 & 26 & 15 & 90 & 59 & 149 & $05.52 \%$ \\
\hline & Furuncle & 02 & 00 & 06 & 04 & 12 & 03 & 20 & 07 & 27 & $01.00 \%$ \\
\hline & Scalp pyoderma & 10 & 06 & 27 & 16 & 17 & 13 & 54 & 35 & 89 & $03.30 \%$ \\
\hline & Boil/carbuncle & 12 & 06 & 56 & 36 & 79 & 33 & 147 & 75 & 222 & $08.23 \%$ \\
\hline & Cellulitis & 02 & 00 & 01 & 00 & 02 & 04 & 05 & 04 & 09 & $00.33 \%$ \\
\hline & Total & 49 & 41 & 255 & 166 & 181 & 99 & 485 & 306 & 791 & $29.33 \%$ \\
\hline \multirow[t]{9}{*}{ Viral } & Molluscum contagiosum & 01 & 00 & 13 & 13 & 03 & 03 & 17 & 16 & 33 & $01.22 \%$ \\
\hline & Chickenpox & 01 & 02 & 13 & 08 & 18 & 23 & 32 & 33 & 65 & $02.41 \%$ \\
\hline & Herpes zoster & 00 & 00 & 01 & 00 & 03 & 01 & 04 & 01 & 05 & $00.18 \%$ \\
\hline & Herpes simplex & 00 & 00 & 00 & 00 & 02 & 03 & 02 & 03 & 05 & $00.18 \%$ \\
\hline & Warts & 00 & 00 & 08 & 10 & 14 & 16 & 22 & 26 & 48 & $01.78 \%$ \\
\hline & Pityriasis rosea & 02 & 00 & 05 & 03 & 19 & 08 & 26 & 11 & 37 & $01.37 \%$ \\
\hline & Handfootmouth disease & 05 & 03 & 09 & 10 & 02 & 00 & 16 & 13 & 29 & $01.07 \%$ \\
\hline & HIV & 00 & 00 & 00 & 00 & 08 & 02 & 08 & 02 & 10 & $00.37 \%$ \\
\hline & Total & 09 & 05 & 49 & 44 & 69 & 56 & 127 & 105 & 232 & $08.60 \%$ \\
\hline \multirow[t]{5}{*}{ Fungal } & Tinea capitis & 06 & 01 & 13 & 05 & 11 & 08 & 30 & 14 & 44 & $01.63 \%$ \\
\hline & Other tinea & 07 & 06 & 23 & 16 & 50 & 14 & 80 & 36 & 116 & $04.30 \%$ \\
\hline & Cutaneous candidiasis & 07 & 07 & 07 & 09 & 07 & 07 & 21 & 23 & 44 & $01.63 \%$ \\
\hline & Pityriasis versicolar & 05 & 02 & 02 & 05 & 16 & 08 & 23 & 15 & 38 & $01.40 \%$ \\
\hline & Total & 25 & 16 & 45 & 35 & 84 & 37 & 154 & 88 & 242 & $08.97 \%$ \\
\hline \multirow[t]{6}{*}{ Parasitic } & Scabies & 29 & 19 & 86 & 63 & 145 & 65 & 260 & 147 & 407 & $15.09 \%$ \\
\hline & Pediculosis capitis & 00 & 00 & 02 & 02 & 01 & 07 & 03 & 09 & 12 & $00.44 \%$ \\
\hline & Papular urticarial & 01 & 05 & 08 & 06 & 02 & 04 & 11 & 15 & 26 & $00.96 \%$ \\
\hline & Insect bite & 02 & 05 & 15 & 05 & 16 & 04 & 33 & 14 & 47 & $00.15 \%$ \\
\hline & Total & 32 & 29 & 111 & 76 & 164 & 80 & 307 & 185 & 492 & $18.25 \%$ \\
\hline & & & & & & & & & & 1757 & $65.19 \%$ \\
\hline
\end{tabular}

Table 5: Pattern of eczema and dermatitis diseases of in >1 month of age group

\begin{tabular}{|l|c|c|c|c|c|c|c|c|c|c|}
\hline \multicolumn{1}{|c|}{ Disease } & \multicolumn{9}{|c|}{ Age group distribution } & \multicolumn{3}{c|}{ Total } & $\begin{array}{c}\text { Grand } \\
\text { total }\end{array}$ & Percentage \\
\hline & $\begin{array}{c}1 \text { month-1 } \\
\text { year }\end{array}$ & \multicolumn{2}{c|}{$\begin{array}{c}>1-6 \\
\text { year }\end{array}$} & \multicolumn{2}{c|}{$\begin{array}{c}>6-14 \\
\text { year }\end{array}$} & & & & \\
\hline & $\mathrm{M}$ & $\mathrm{F}$ & $\mathrm{M}$ & $\mathrm{F}$ & $\mathrm{M}$ & $\mathrm{F}$ & $\mathrm{M}$ & $\mathrm{F}$ & & \\
\hline Atopic dermatitis & 12 & 10 & 18 & 19 & 12 & 10 & 42 & 39 & 81 & $03.00 \%$ \\
\hline Seborrheic dermatitis & 08 & 06 & 11 & 13 & 09 & 20 & 28 & 39 & 67 & $02.48 \%$ \\
\hline Pityriasis alba & 06 & 04 & 16 & 12 & 23 & 08 & 45 & 24 & 69 & $02.55 \%$ \\
\hline $\begin{array}{l}\text { Irritant contact } \\
\text { dermatitis }\end{array}$ & 00 & 00 & 02 & 04 & 03 & 03 & 05 & 07 & 12 & $00.44 \%$ \\
\hline Perianal dermatitis & 01 & 00 & 03 & 01 & 01 & 01 & 05 & 02 & 07 & $00.25 \%$ \\
\hline Diaper dermatitis & 01 & 00 & 00 & 00 & 00 & 00 & 01 & 00 & 01 & $00.03 \%$ \\
\hline Pompholyx & 00 & 00 & 01 & 00 & 00 & 00 & 00 & 01 & 01 & $00.03 \%$ \\
\hline Paedrus dermatitis & 00 & 00 & 02 & 02 & 03 & 03 & 05 & 05 & 10 & $00.37 \%$ \\
\hline Eczema & 02 & 00 & 09 & 13 & 34 & 18 & 45 & 31 & 76 & $02.82 \%$ \\
\hline & 30 & 20 & 62 & 64 & 85 & 63 & 177 & 147 & 324 & $12.01 \%$ \\
\hline
\end{tabular}

\section{Discussion}

The present study was conducted from March 2015 to February 2016 at tertiary care center, Gujarat during which 2456 cases were included. Patients were divided in four category which include neonate ( $<1$ month), infant ( $>1$ month- 1 year), preschool child $(>1$ year- 6 year) and school child (>6year- 14 year). Amongst all age groups, most common age group was school child (44.09\%, $\mathrm{n}=1083)$ followed by preschool child 
(41.57\%, $\mathrm{n}=1021)$, infant $(12.09 \%, 297)$ and least common was neonate $(02.23 \%, \mathrm{n}=55)$ (Table 1$)$.

Out of the 2456 patients, 1454 (59.20\%) patients were males and $1002(40.80 \%)$ were females. The male to female ratio in our study was $1.43: 1$ which was comparable to study done by Jawade et $\mathrm{al}^{6}$ where M:F ratio was 1.40:1 and lesser reported by Bisht et $\mathrm{al}^{3}$ (1.30:1).

In our study, maximum cases were in age group was 6-14 years which was $44.09 \%$, whereas in study by Jawade et al, ${ }^{6}$ it was $2^{\text {nd }}$ most common age group at $33 \%$. In their study most common age group was 1-6 years $(46.32 \%)$ which was comparable to our study (41.57\%).

Amongst neonatal skin diseases, physiological skin conditions constituted $40 \%$ in our study which was comparable to study done by Jawade et al (39.69\%), ${ }^{6}$ in other studies like Nobbay and Chakrobarthy ${ }^{7}(69 \%)$, Baruah et $\mathrm{al}^{8}(93 \%)$, Kulkarni and $\operatorname{Singh}^{9}(72 \%)$, and Patel $(78 \%) .{ }^{10}$

In our study, infectious diseases were most common (65.19\%) which was comparable to study done by Jawade et $\mathrm{al}^{6}(56.40 \%)$. In their study, Hassan et $\mathrm{al}^{11}$ found only $29.40 \%$ disorder of infectious etilogy while Dogra and Kumar ${ }^{12}$ found only $11.4 \%$. Different studies across India have found infectious and infestations contributed $35.6 \%$ to $85.2 \%$ of all paediatric dermatoses. ${ }^{4,5,13,14}$

Eczema and dermatitis group constituted $12.02 \%$ in our study which was comparable to Jawade et $\mathrm{al}^{6}$ $(15.61 \%)$ and Hassan et $\mathrm{al}^{11}(12.0 \%)$. In the study conducted by Dogra \& Kumar, ${ }^{12}$ incidence of dermatitis was only $5.2 \%$.

Pigmentary disorders were $2.15 \%$ in our study which was comparable to study of Thappa ${ }^{14}$ (3.16\%) and Jawade et $\mathrm{al}^{6}(2.80 \%)$ while higher incidence was found in study of Patel et $\mathrm{al}^{10}(11.48 \%)$, Hassan et al ${ }^{11}$ (14.10\%), and Ben Saif \& Al Shehab ${ }^{1}$ (8.9\%).

Nutrional disorders were $2.67 \%$ in our study which was supported by study done by Jawade et $\mathrm{al}^{6}(3.80 \%)$ while in study done by Hassan et al, ${ }^{11}$ it was seen only in $0.4 \%$. In study done by Negi et al, ${ }^{15}$ it was $17.5 \%$. Our study was done in urban set up as compared to Negi et ${ }^{15}$ al which was done in rural area accounting for difference in incidence.

In our study, Papulosquamous disorder was $1.89 \%$ which was comparable to Hassan et al (1.8\%). In the study done by Jawade et al, ${ }^{6}$ it was $9.10 \%$.

Keratinizing disorder was $2.00 \%$ in our study which was supported by study done by Jawade et $\mathrm{al}^{6}$ (2.58\%) and Hassan et $\mathrm{al}^{11}(3.2 \%)$

Vesiculobullous disorder $0.04 \%$ in our study was comparable to study by Jawade et $\mathrm{al}^{6}$ and Hassan et a ${ }^{11}$ ( $0.33 \%$ and $0.46 \%$ respectively).

Incidence of Nevoid and other development $(1.41 \%)$ disorders in our study was comparable to the study done by Hassan et al ${ }^{11}(1.1 \%)$.
In our study, amongst infectious diseases, bacterial infections were maximum $(29.33 \%)$ followed by parasitic infections (18.25\%). Viral and fungal infections were $15.09 \%$ and $08.60 \%$ respectively. In the study done by Jawade et al, ${ }^{6}$ parasitic infections were maximum $(25.73 \%)$. Bacterial, fungal, and viral diseases were $13.94 \%, 8.65 \%$, and $8.08 \%$ respectively. Among bacterial infection, impetigo (10.94\%), boil and cabuncle (8.23) and scalp pyoderma (3.30\%) were most common. However in study done by Jawade et al, ${ }^{6}$ impetigo and pyoderma were $5.96 \%$ and $5.62 \%$ respectively. The study done by Vikash Bhatia et al ${ }^{16}$ found $16.1 \%$ cases of pyoderma which was higher than our study and Jawade et al. ${ }^{6}$

Scabies (15.09\%) was most common parasitic infestation. In other studies conducted in various places, incidence rate of scabies range from $5.1 \%$ to $24.49 \%$. $^{4-}$ $6,12-14,17,16$ In our study, incidence of pediculosis capitis was $0.44 \%$ which was similar to study by Jawade et $\mathrm{al}^{6}$ $(0.45 \%)$ and Rao et al $(0.5 \%){ }^{17}$

In our study, most common viral infection was chickenpox $(2.41 \%)$ followed by warts $(1.78 \%)$ and molluscum contagiosum (1.22\%). In study done by Jawade et al, ${ }^{6}$ molluscum contagiosum $(5.39 \%)$ was most common followed by viral warts $(1.35 \%)$. In study done by Patel et $\mathrm{al}^{10}$ highest incidence were warts (1.53\%).

Among fungal infections, in our study tinea corporis was $4.30 \%$ and tinea capitis was $1.63 \%$. However in study done by Jawade et al, ${ }^{6}$ tinea capitis and tinea corporis were $4.49 \%$ and $1.46 \%$ respectively. The incidence of pityriasis versicolar and cutaneous candidiasis in our study were similar to the study of Jawade et al. ${ }^{6}$

The incidence of atopic dermatitis in our study was $3.37 \%$ which was similar to study by Jawade et al ${ }^{6}$ $(4.27 \%)$. Studies conducted all over word found incidence rate of atopic dermatitis ranges from $3 \%$ to $28 \% .{ }^{18-21}$ Pityriasis alba (2.87\%) in our study, whereas study done by Jawade et al, ${ }^{6}$ it was $4.16 \%$. Incidence of seborrheic dermatitis was $2.79 \%$ in our study. Similar incidence were found in Ben Saif \& $\mathrm{Al}$ Shehab (3.4\%), ${ }^{1}$ Jawade et $\mathrm{al}^{6}(3.60 \%)$ and Patel et $\mathrm{al}^{10}(1.82 \%)$.

Incidence of pityriasis rosea in our study was $1.37 \%$ which was comparable to study by Ben Saif \& Al Shehab ${ }^{1}(2.1 \%)$ and Jawade et $\mathrm{al}^{6}(3.15 \%)$. Pityriasis Rosea accounts for $2 \%$ outpatient visits in dermatology..$^{22}$

Incidence of lichen planus in our study was $0.87 \%$. Higher incidence was found in study by Samman $(2 \%),{ }^{23}$ Handa \& Sahoo (2\%), ${ }^{24}$ Jawade et al $(2.58 \%),{ }^{6}$ Kumar \& Collegue et al $(11.2 \%)^{25}$ and Luis Montoya $(10.2 \%) .{ }^{26}$ Incidence of lichen nitidus $(0.33 \%)$, lichen spinulous $(0.08 \%)$, lichen striatus $(0.41 \%)$ were comparable to Jawade et $\mathrm{al}^{6}(1.12 \%, 0.67 \%$, and $0.11 \%$ respectively). 
Incidence of psoriasis was $0.49 \%$ in our study. Similar observation reported by Jawade et $a l,{ }^{6}$ Rao et $\mathrm{al}^{17}$ and Sardana $\mathrm{K}^{5}$ in their studies.

Incidence of icthyosis $(0.33 \%)$ and keratoderma (1.54\%) was comparable to study done by Jawade et $\mathrm{al},{ }^{6}$ Ghosh et $\mathrm{al}^{27}$ and Porter et al. ${ }^{28}$

Bullous disorder $(0.04 \%)$ in our study was comparable to study done by Jawade et $\mathrm{al}^{6}(0.33 \%)$.

Urticarial was found in $3.29 \%$ of cases in our study which was comparable to study done by Jawade et $\mathrm{al}^{6}$ $(1.35 \%)$ and other study. In our study, haemangioma was found in $0.54 \%$ of cases which was similar to study done by Jawade et $\mathrm{al}^{6}(0.79 \%)$.

Miliria (5.03\%) was found in our study which was higher than study done by Jawade et $\mathrm{al}^{6}(1.12 \%)$ whereas other appendageal disorder such as alopecia aerate $(0.83 \%)$, milia $(0.41 \%)$ and acne $(0.74 \%)$ in our study was comparable to study done by Jawade et $\mathrm{al}^{6}$ $(0.79 \%, 0.79 \%$ and $0.45 \%$ respectively).

\section{References}

1. Saif GA, Al Shehab SA. Pattern of childhood dermatoses at a teaching hospital of Saudi Arabia. International Journal of Health Sciences. 2008;2(2):63.

2. Schachner LA, Hansen RG. Preface. In: Schachner LA, Hansen RG, editors. Pediatric dermatology. 2nd ed. New York: Churchill Livingstone; 1995. p. 9.

3. Bisht JS, Rana SK, Kumari N, Aggarwal B, Mehta A, Singh R. Pattern of dermatoses in preschool children in a teaching hospital in Uttarakhand, India. Indian Journal of Paediatric Dermatology. 2015;16(4):198.

4. Sharma NK, Garg BK, Goel M. Pattern of Skin Diseases in Urban School Children. Indian Journal of Dermatology, Venereology and Leprology. 1986;52(6):330.

5. Sardana K, Mahajan S, Sarkar R, Mendiratta V, Bhushan $\mathrm{P}$, Koranne RV, Garg VK. The spectrum of skin disease among Indian children. Pediatric Dermatology. 2009;26(1):6-13.

6. Jawade SA, Chugh VS, Gohil SK, Mistry AS, Umrigar DD. A clinico-etiological study of dermatoses in pediatric age group in tertiary health care center in South Gujarat region. Indian Journal of Dermatology. 2015;60(6):635.

7. Nobbay B, Chakrabarty N. Cutaneous manifestation in the new-born. Indian Journal of Dermatology, Venereology, and Leprology. 1992;58:69-72.

8. Baruah CM, Bhat V, Bhargava R, Garg RB. Prevalence of dermatoses in the neonates in Pondichery. Indian Journal of Dermatology, Venereology, and Leprology. 1991;57(1):25.

9. Kulkarni ML, Singh R. Normal variants of skin in neonates. Indian Journal of Dermatology, Venereology, and Leprology. 1996;62(2):83.

10. Patel JK, Vyas AP, Berman B, Vierra M. Incidence of childhood dermatosis in India. Skinmed. 2009;8(3):13642.

11. Hassan I, Anwar P, Bilquis S, Nabi S, Rasool F, Munshi I. Comparison of dermatoses seen in community health camps and a tertiary care centre in Kashmir. Indian Journal of Dermatology, Venereology, and Leprology. 2014;80(3):214.

12. Dogra S, Kumar B. Epidemiology of skin diseases in school children: a study from northern India. Pediatric dermatology. 2003;20(6):470-3.
13. Kraning KK, Odland GF. Prevalence, morbidity, and cost of dermatological diseases. J Invest Dermatol. 1979;73(5 Pt 2):395-401.

14. Thappa DM. Common skin problems. Indian Journal of Pediatrics. 2002;69(8):701-6.

15. Negi KS, Kandpal SD, Parsad D. Pattern of skin diseases in children in Garhwal region of Uttar Pradesh. Indian Pediatrics. 2001;38(1):77-80.

16. Bhatia V. Extent and pattern of paediatric dermatoses in rural areas of central India. Indian Journal of Dermatology, Venereology, and Leprology. 1997;63(1):22.

17. Rao SG, Kumar P, Kuruvilla M. Prevalence of various dermatoses in school children. Indian Journal of Dermatology, Venereology, and Leprology. 1999;65(3):126.

18. Federman DG, Reid MC, Feldman SR, Greenhoe J, Kirsner RS. The primary care provider and the care of skin disease: the patient's perspective. Archives of dermatology. 2001;137(1):25-9.

19. Speare R, Buettner PG. Head lice in pupils of a primary school in Australia and implications for control. International journal of dermatology. 1999;38(4):285-90.

20. Bowker NC, Cross KW, Fairburn EA, Wall M. Sociological implications of an epidemiological study of eczema in the city of Birmingham. British Journal of Dermatology. 1976;95(2):137-44.

21. Horn R. The pattern of skin diseases in general practice. Dermatology Practice. 1986:2:14-9.

22. González LM, Allen R, Janniger CK, Schwartz RA. Pityriasis rosea: an important papulosquamous disorder. International Journal of Dermatology. 2005;44(9):75764.

23. Samman PD. Lichen planus. An analysis of 200 cases. Transactions of the St. John's Hospital Dermatological Society. 1961;46:36.

24. Handa S, Sahoo B. Childhood lichen planus: a study of 87 cases. International journal of dermatology. $2002 \mathrm{Jul}$ 1;41(7):423-7.

25. Kumar V, Garg BR, Baruah MC, Vasireddi SS. Childhood lichen planus (LP). The Journal of dermatology. 1991;20(3):175-7.

26. Luis- Montoya P, Domínguez- Soto L, Vega- Memije E. Lichen planus in 24 children with review of the literature. Pediatric Dermatology. 2005;22(4):295-8.

27. Ghosh SK, Saha DK, Roy AK. A clinico-aetiological study of dermatoses in paediatric age group. Indian Journal of Dermatology. 1995;40(1):29.

28. Porter MJ, Mack RW, Chaudhary MA. Pediatric skin disease in Pakistan: A study of three Punjab villages. International Journal of Dermatology. 1984;23(9):613-6.

How to cite this article: Prajapati KM, Padhiar B. A study of paediatric dermatoses in tertiary care center, Gandhinagar, Gujarat. Ind J Clin Exp Dermatol. 2018;4(3):179-184. 